\title{
Deformidade facial decorrente de tumor odontogênico: Relato de caso
}

\author{
Facial deformity arising from odontogenic tumor: Case report \\ Deformidad facial por tumor odontógeno: Reporte de caso
}

Recebido: 19/04/2021 | Revisado: 26/04/2021 | Aceito: 30/04/2021 | Publicado: 14/05/2021

Leandro de Menezes Galindo Filho
ORCID: https://orcid.org/0000-0002-7415-7495
Universidade de Pernambuco, Brasil
E-mail: leomenezesfilho@gmail.com
Matheus Eduardo da Silva Paz
ORCID: https://orcid.org/0000-0002-5491-1980
Universidade de Pernambuco, Brasil
E-mail: matheuspaaz1 @ gmail.com
Nathália Gomes Buarque Rodrigues
ORCID: https://orcid.org/0000-0003-2713-1011
Universidade de Pernambuco, Brasil
E-mail:buarquenath@gmail.com
Cauê Fontan Soares
Hospital Getúlio Vargas, Brasil
ORCID: https://orcid.org/0000-0002-9392-4530
E-mail: caue_fontan_@ @otmail.com
Jose Romero Souto de Souza Junior
ORCID: https://orcid.org/0000-0002-3655-8466
Universidade de Pernambuco, Brasil
E-mail: romerosouto@ @otmail.com
Joaquim Celestino da Silva Neto
ORCID: https://orcid.org/0000-0001-6660-4948
Universidade de Pernambuco, Brasil
E-mail: joaquimcelestino@ @otmail.com.br

\begin{abstract}
Resumo
Os tumores odontogênicos (TO) formam um grupo de lesões com diferentes tipos histológicos e aspectos clínicos, podendo variar desde hamartomas até lesões malignas, apresentando, assim, uma variedade de manifestações clínicas. Apesar dos comportamentos clínicos distintos, os TO alteram a morfologia dos tecidos afetados gerando um acometimento estético e funcional ao paciente, que sem tratamento, pode tornar incapacitante a depender da agressividade do tumor. O objetivo do trabalho é relatar um caso de uma paciente com deformidade facial decorrente de um ameloblastoma em mandíbula tratado com enucleação. Relato de caso: Paciente jovem, com queixas de estalos em mandíbula após se alimentar e deformidade facial progressiva. Ao exame físico observou-se aumento de volume extraoral em ângulo mandibular direito, intraoral em mucosa jugal e tecido retromolar causando mordedura da mucosa. Ao exame radiográfico foi percebido uma imagem radiolúcida, unilocular, abrangendo a região de corpo mandibular até ramo mandibular. A paciente foi submetida a punção, biópsia e instalação de dispositivo de descompressão na região, que permaneceu em posição por 8 meses, sem o sucesso esperado. Com o diagnóstico de ameloblastoma, foi optado por realizar enucleação e curetagem da lesão, que apresentou boa eficácia terapêutica devolvendo à paciente o padrão facial prévio e restaurando as funções mastigatórias. Conclusão: A repercussão dos tumores odontogênicos na qualidade de vida dos pacientes é significativa e sua terapêutica deve ser estabelecida levando sempre em consideração o tipo do tumor, sua localização e agressividade, visando restabelecer as funções do sistema estomatognático da melhor forma possível.
\end{abstract}

Palavras-chave: Tumores odontogênicos; Qualidade de vida; Terapêutica.

\begin{abstract}
Odontogenic tumors (OT) form a group of lesions with different histological types and clinical aspects, which can vary from hamartomas to malignant lesions, thus presenting a variety of clinical manifestations. Despite the different clinical behaviors, OT change the morphology of the affected tissues, generating an aesthetic and functional impairment to the patient, which, without treatment, can make it incapacitating depending on the aggressiveness of the tumor. The objective of the work is to report a case of a patient with facial deformity due to an ameloblastoma in the mandible treated with enucleation. Case report: young patient, with complaints of snapping jaws after eating and progressive facial deformity. On physical examination, there was an increase in extraoral volume at the right, intraoral mandibular angle in the cheek mucosa and retromolar tissue causing bitening of the mucosa. Radiographic examination revealed a radiolucent, unilocular image, covering the region of the mandibular body up to the mandibular ramus. The patient
\end{abstract}


underwent puncture, biopsy and installation of a decompression device in the region, which remained in position for 8 months, without the expected success. With the diagnosis of ameloblastoma, it was decided to perform enucleation and curettage of the lesion, which showed good therapeutic efficacy by returning the patient to the previous facial pattern and restoring masticatory functions. Conclusion: The impact of odontogenic tumors on the quality of life of patients is significant and their therapeutics must be established taking into account the type of tumor, its location and aggressiveness, in order to reestablish the functions of the stomatognathic system in the best possible way.

Keywords: Odontogenic tumors; Quality of life; Therapeutics.

\section{Resumen}

Los tumores odontogénicos (TO) forman un grupo de lesiones con diferentes tipos histológicos y aspectos clínicos, que pueden variar desde hamartomas hasta lesiones malignas, presentando así una variedad de manifestaciones clínicas. A pesar de los diferentes comportamientos clínicos, los TO cambian la morfología de los tejidos afectados, generando deterioro estético y funcional en el paciente, que, sin tratamiento, puede hacerlo incapacitante para depender de la agresividad del tumor. El objetivo del trabajo es reportar el caso de un paciente con deformidad facial por ameloblastoma en mandíbula tratado con enucleación. Caso clínico: Paciente joven, con quejas de mandíbulas chasqueantes después de comer y deformidad facial progresiva. El examen físico mostró un aumento del volumen extraoral en el ángulo mandibular derecho, intraoral en la mucosa de la mejilla y tejido retromolar provocando una picadura de la mucosa. El examen radiográfico reveló una imagen unilocular radiolúcida, que cubría la región del cuerpo mandibular hasta la rama mandibular. El paciente fue sometido a punción, biopsia e instalación de un dispositivo de descompresión en la región, que permaneció en posición durante 8 meses, sin el éxito esperado. Con el diagnóstico de ameloblastoma se decidió realizar la enucleación y legrado de la lesión, que mostró buena eficacia terapéutica al devolver al paciente al patrón facial anterior y restaurar las funciones masticatorias. Conclusión: La repercusión de los tumores odontogénicos en la calidad de vida de los pacientes es significativa y su tratamiento debe establecerse teniendo en cuenta el tipo de tumor, su ubicación y agresividad, con el objetivo de restablecer de la mejor manera posible las funciones del sistema estomatognático.

Palabras clave: Tumores odontogénicos; Calidad de vida; Terapéutica.

\section{Introdução}

Os tumores odontogênicos (TO) englobam um conjunto de lesões com comportamentos clínicos e histopatológicos heterogêneos (da Silva et al., 2016; Neville, Damm, Allen \& Chi, 2016). Eles possuem em sua maioria alterações radiográficas envolvendo elementos dentários, geralmente acompanhados de sintomatologia crescente ao decorrer da evolução da lesão. Porém, o aumento de volume observado acarretando assimetria facial é um dos achados clínicos mais significativos por ser o principal motivo da busca por atendimento especializado (Santosh \& Ogle, 2020).

O ameloblastoma é o tumor odontogênico clinicamente significativo mais comum, porém o segundo mais prevalente entre todos, perdendo apenas para o odontoma (Chae, Smoll, Hunter-Smith \& Rozen, 2015). Constitui de 1\% e 3\% de todos os tumores e cistos dos ossos maxilares, ocorrendo geralmente na mandíbula, sendo a porção posterior mais afetada (Santosh \& Ogle, 2020; Shi, Ng, Kwa \& Sim, 2020). Assim como a maioria dos TO, o aumento de volume indolor nos ossos gnáticos é seu principal achado clínico (McClary et al., 2016) e não possui predileção por sexo. Apesar de ser um tumor benigno, o ameloblastoma apresenta alto potencial de destruição local com crescimento infiltrativo (Santana et al., 2019).

De acordo com a atual classificação de tumores odontogênicos da Organização Mundial da Saúde (OMS) de 2017, o ameloblastoma é dividido em três categorias: (a) ameloblastoma convencional, (b) ameloblastoma unicístico, e (c) ameloblastoma extraósseo ou periférico (de Medeiros, da Silva, Santos, Pinto, \& de Souza, 2018; Shi et al., 2020). Cada categoria apresenta tratamento e prognóstico diferentes, por isso, o exame histopatológico é obrigatório para a confirmação do diagnóstico e escolha ideal do tratamento (Santana et al., 2019; Santosh \& Ogle, 2020).

Os TO são tratados por uma ampla gama de opções cirúrgicas que vão desde a tratamentos mais conservadores como descompressão e enucleação até ressecção cirúrgica. Alguns desses tumores têm tendência à recorrência, portanto, o acompanhamento pós-cirúrgico periódico se faz necessário. O tratamento adequado visa não só remover a patologia como também devolver qualidade de vida e diminuir a morbidade do paciente (Santosh \& Ogle, 2020; Slusarenko da Silva, Tartaroti, Sendyk, Deboni, \& Naclério-Homem, 2018). 
O objetivo do presente trabalho é expor um caso clínico de uma paciente jovem apresentando deformidade facial significativa causada por um tumor odontogênico.

\section{Metodologia}

O presente trabalho trata-se de um estudo observacional descritivo de um relato de caso. Para complementar a composição da discussão uma revisão de literatura foi realizada por meio da pesquisa de artigos científicos que tenham relevância e que abordem de forma semelhante o caso descrito. As pesquisas foram feitas através do portal PubMed e da base de dados LILACS dos últimos 13 anos. Por se tratar de um Relato de Caso Clínico, não houve necessidade de aprovação pelo Comitê de Ética em Pesquisa da instituição.

\section{Relato de Caso}

Paciente, 30 anos, xantoderma compareceu ao serviço de Cirurgia e Traumatologia Bucomaxilofacial do Hospital Getúlio Vargas/PE com queixa principal de "uma mordida em uma cana que resultou em um estalo". Durante exame físico, observou-se aumento de volume indolor em corpo, ângulo e ramo da mandíbula do lado direito, causando deformidade significativa na face da paciente (Figura 1-A, 1-B). Uma vez feito exame radiográfico, constatou-se uma imagem radiolúcida estendendo-se desde a região posterior do corpo mandibular direito, na altura do elemento 47, até o ramo mandibular direito, na altura da língula mandibular, com integridade da borda posterior da mandíbula (Figura 1-C). Ao exame físico intraoral, foi possível observar um aumento de volume responsável pela ocorrência de mordidas na mucosa jugal da paciente, evidenciado por marcas hiperemiadas na região de mucosa jugal e trígono retromolar direito (Figura 1-D). 
Figura 1. Paciente em vista frontal em A e B apresentando tumoração facial na região mandibular direita. Imagem intraoral destacando aumento de volume em C. Imagem radiográfica panorâmica mostrando a reabsorção óssea na região de corpo, ângulo e ramo mandibular do lado direito em $\mathrm{D}$.
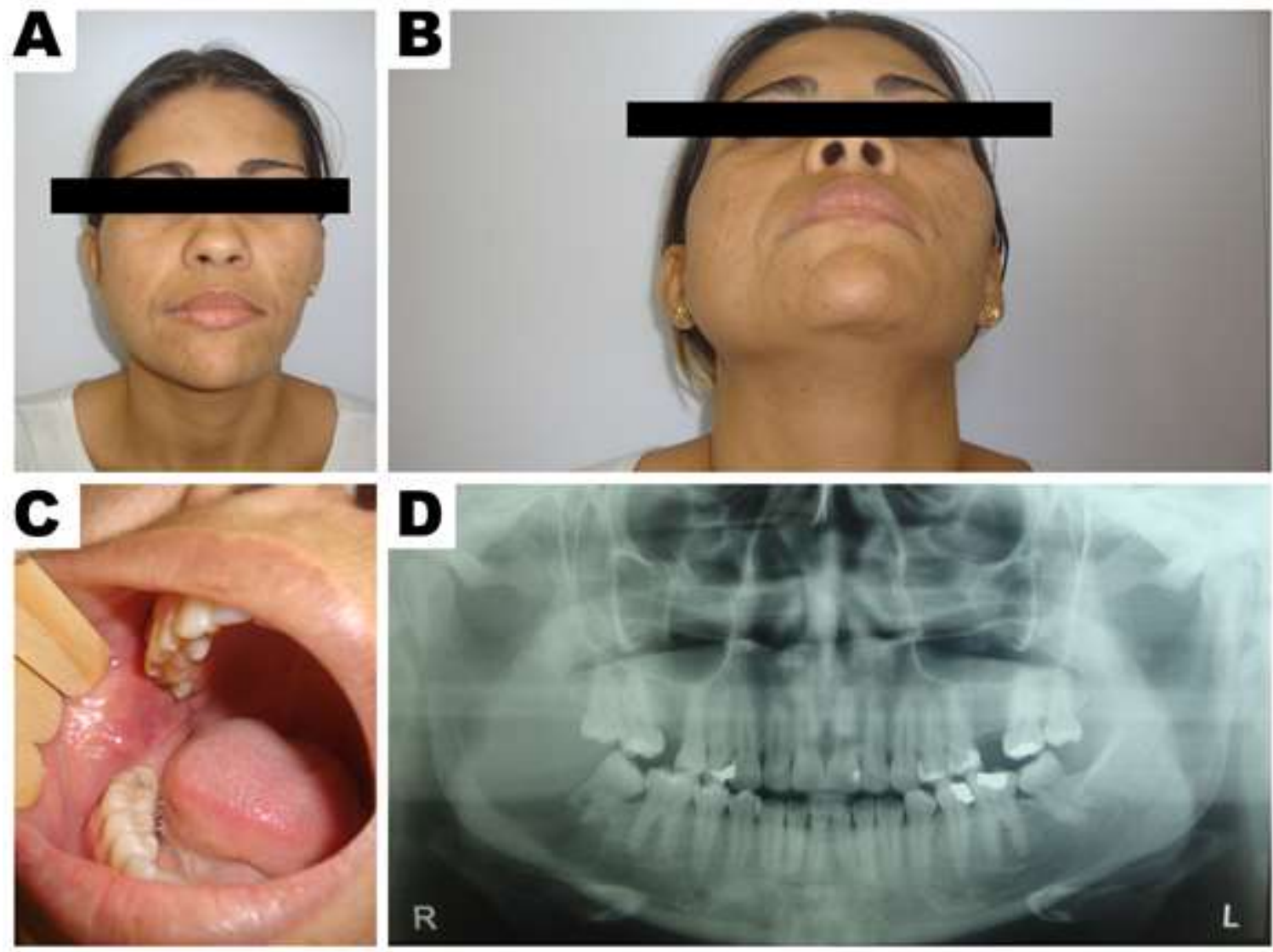

Fonte: Autores.

A paciente foi submetida a biópsia incisional (Figura 2-A) e punção (Figura 2-B), a qual foi retirado cerca de $25 \mathrm{ml}$ de secreção escura e sanguinolenta (Figura 2-C), seguida da instalação de um dispositivo para descompressão (Figura 2-D). O material foi encaminhado ao histopatológico, no qual a microscopia apontava uma camada de células basais dispostas em paliçada, com núcleos apresentando polaridade invertida. As camadas suprabasais se apresentavam com células mais espaçadas, conferindo um aspecto mixóide, sendo o diagnóstico compatível com ameloblastoma e sem sinais de malignidade. 
Figura 2. Dois fragmentos medindo 1,5 x 1,3 x 0,5 cm retirados para a biópsia em A, processo de punção em B e líquido retirado da punção em C. Em D, dispositivo para o procedimento de descompressão.
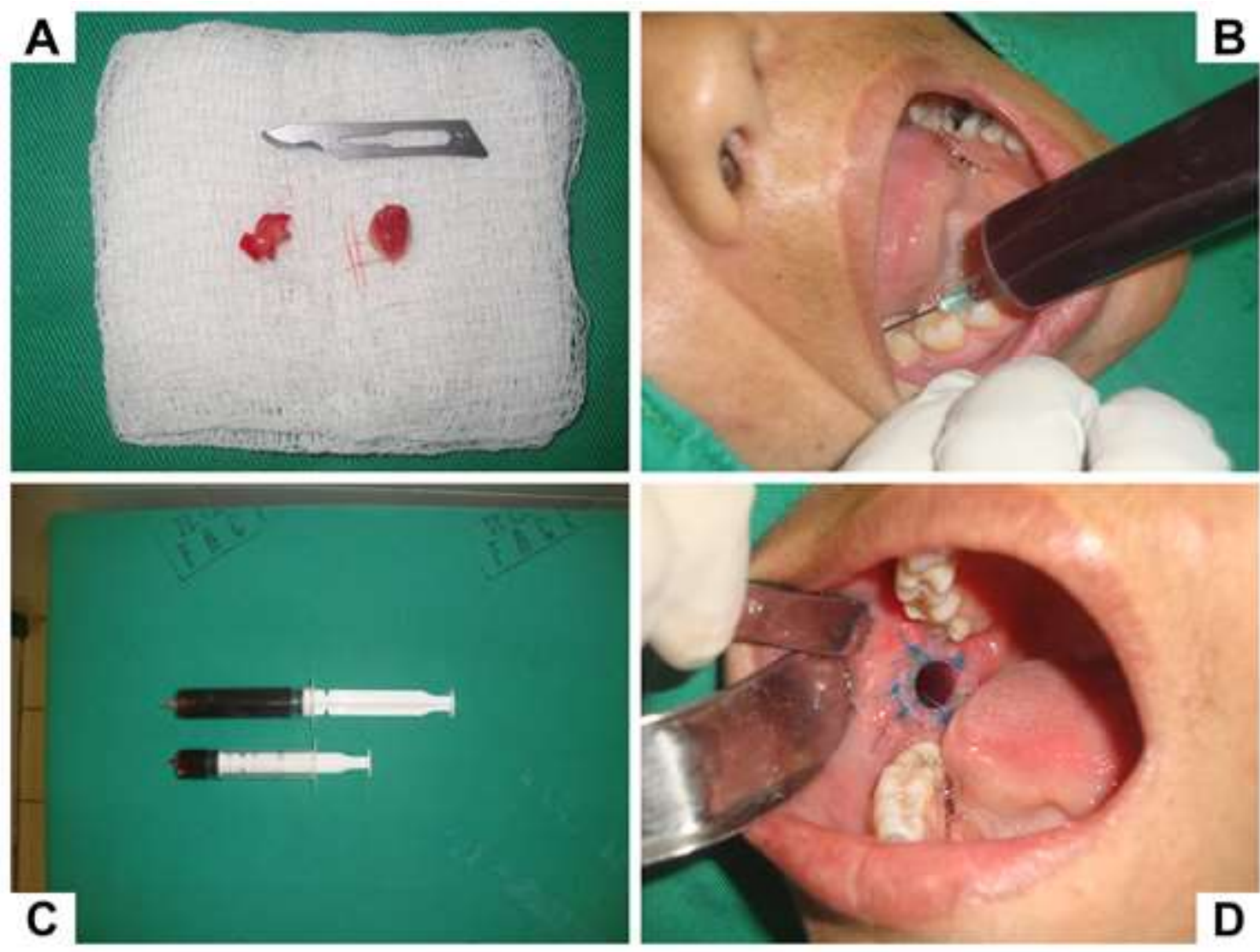

Fonte: Autores.

A paciente permaneceu com o dispositivo de descompressão e foi acompanhado sua evolução. Cerca de 8 meses após a primeira conduta foi averiguado, a partir de radiografia e tomografia, que o processo de descompressão não estava mais sendo satisfatório com regressão mínima da lesão (Figura 3 e Figura 4). Foi optado, então, uma abordagem cirúrgica, constituída por enucleação da lesão (Figura 5-A) e curetagem da cavidade (Figura 5-B). 
Figura 3. Radiografia panorâmica feita após 8 meses de acompanhamento, sem evolução satisfatória quanto a neoformação óssea e permanência da radiolucidez na região de corpo de mandíbula.

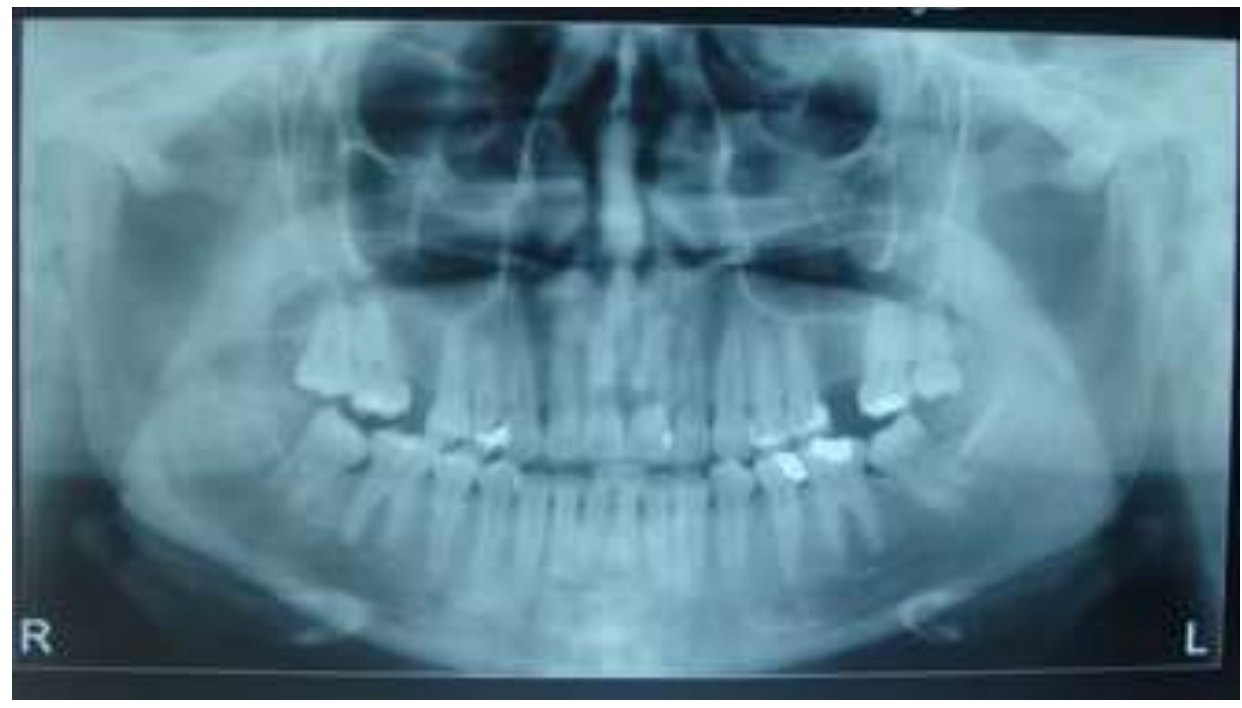

Fonte: Autores.

Figura 4. Imagem da tomografia computadorizada, corte axial, mostrando a discrepância entre o lado esquerdo, sem a lesão, e o lado direito, com a lesão osteolítica. A lesão se vê representada por um espaço de hipodensidade na região próxima ao elemento 47, demonstrando expansão de cortical óssea e pouca regressão da lesão.

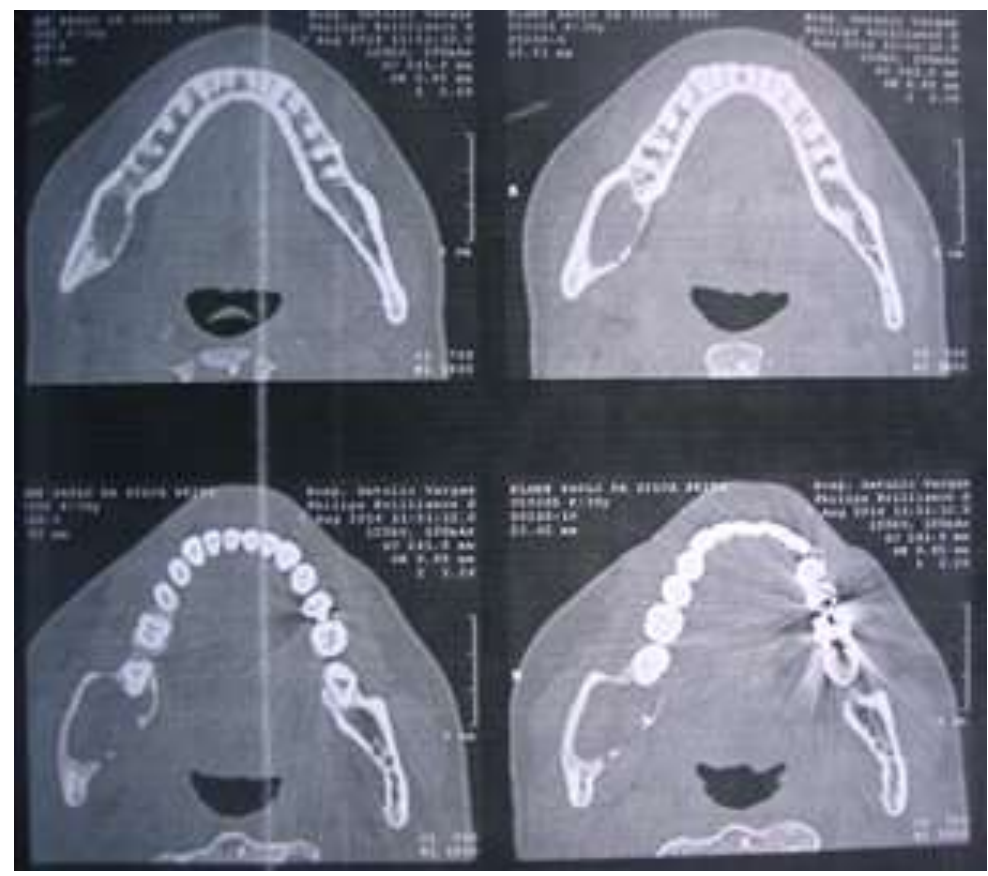

Fonte: Autores.

O procedimento foi realizado e, mesmo já havendo diagnóstico prévio da primeira biópsia incisional, a nova peça curetada foi encaminhada ao histopatológico (Figura 5-C), a fim de confirmar o diagnóstico. Paralelamente ao procedimento foi retirado também o elemento 47 envolvido na lesão (Figura 5-E), e o aparelho de descompressão (Figura5-D). O segundo resultado foi condizente com o primeiro, firmado o caso de Ameloblastoma plexiforme. 
Research, Society and Development, v. 10, n. 4, e48110514077, 2021

(CC BY 4.0) | ISSN 2525-3409 | DOI: http://dx.doi.org/10.33448/rsd-v10i5.14077

Figura 5. O procedimento iniciou-se pela enucleação da cavidade A, seguida de curetagem B. Em C, o material curetado e em E o dente extraído pois estava relacionado a lesão. E em D o dispositivo usado previamente durante o processo de descompressão.
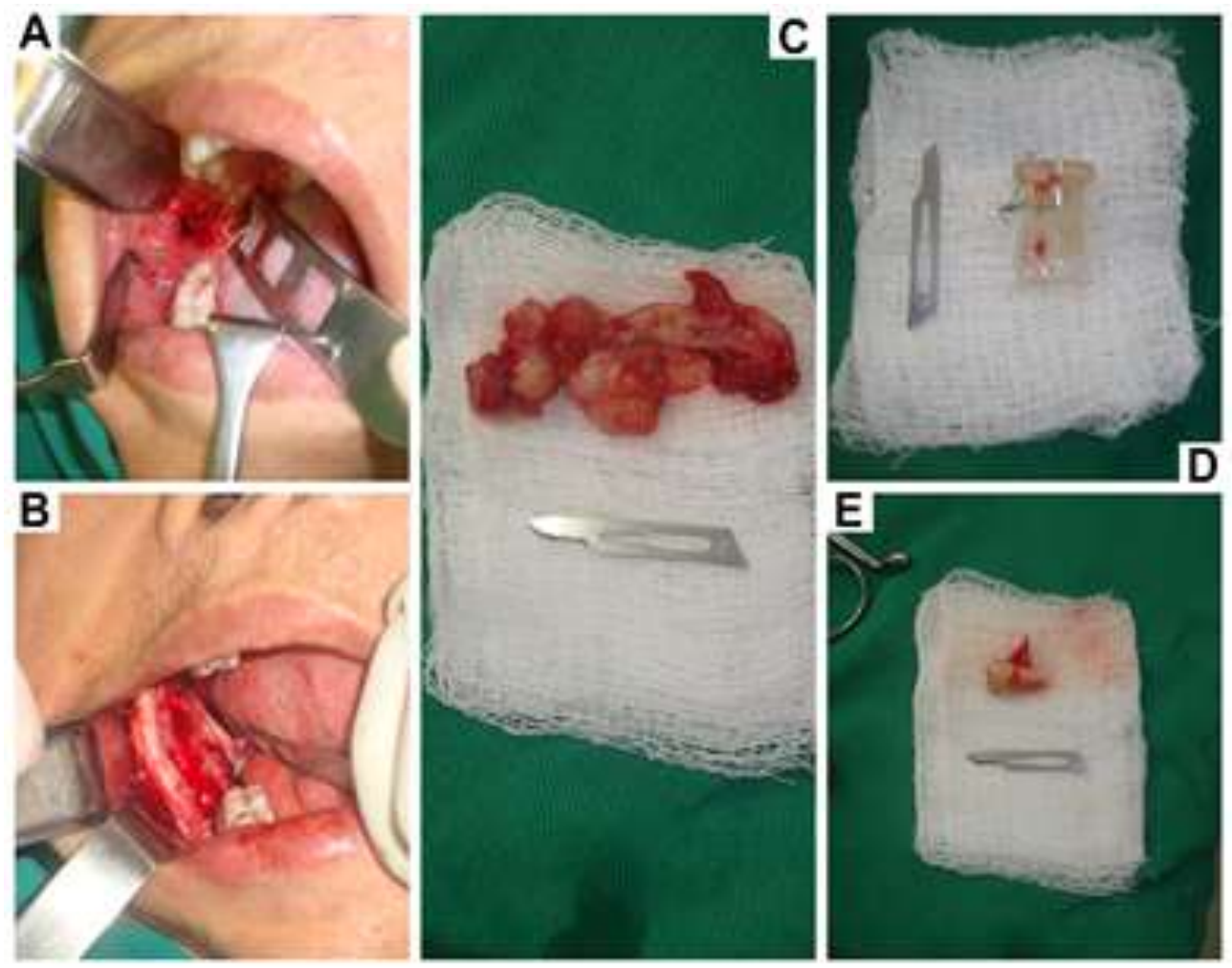

Fonte: Autores.

A paciente permanece sendo acompanhada, até o momento com 1 ano e 2 meses de pós-operatório, sem sinais de recidiva. Além disso, houve regressão do aumento de volume e restabelecimento da estética e função (Figura 6). 
Figura 6. Radiografia de acompanhamento feita 1 ano e 2 meses após a enucleação e curetagem, percebe-se uma melhor situação do corpo mandibular devido a neoformação óssea em A. Em B, a visão frontal com a harmonia facial estabelecida. E em C, a visão interna da cavidade bucal, não mais com aumento de volume.

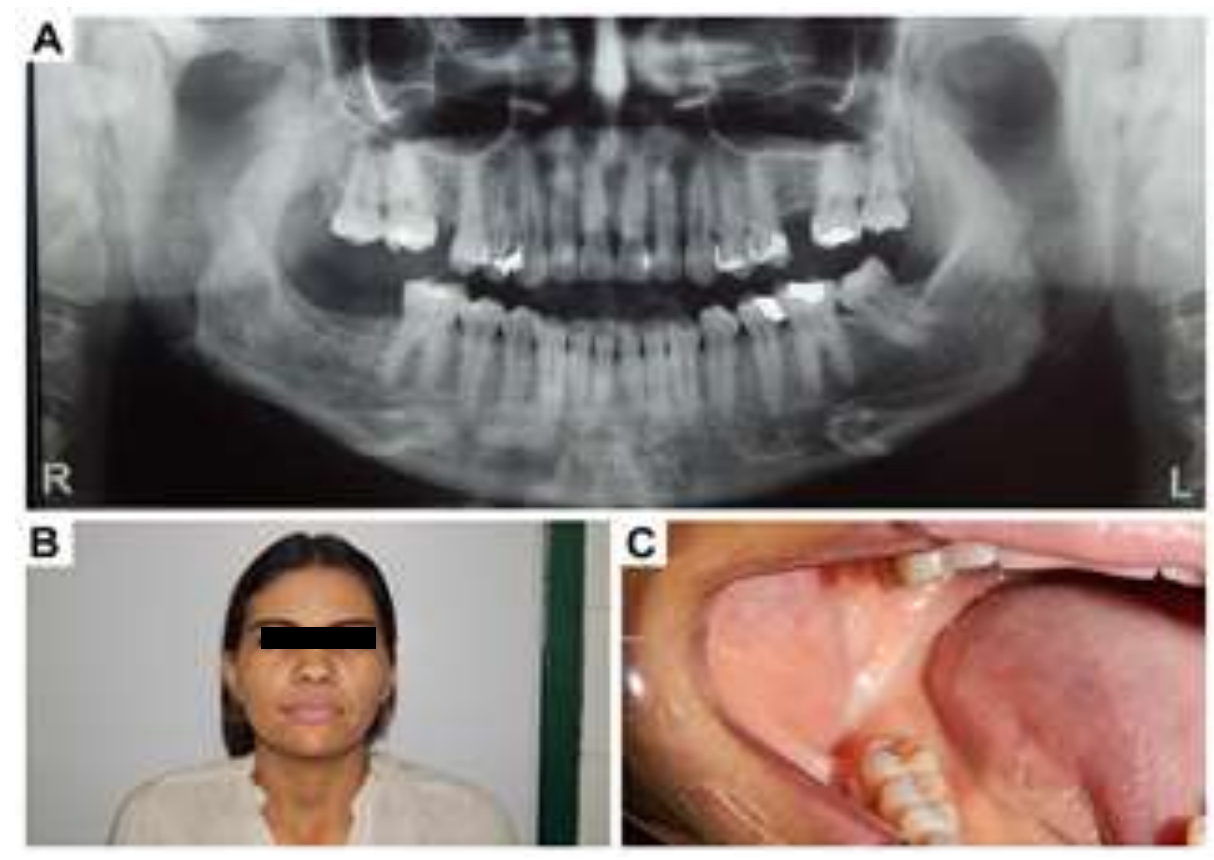

Fonte: Autores.

\section{Resultados e Discussão}

Em 2017 a OMS classificou o ameloblastoma como um tumor odontogênico benigno de origem epitelial e dividiu os ameloblastomas em 3 tipos, sendo eles o ameloblastoma convencional, ameloblastoma unicístico e ameloblastoma periférico ou extra ósseo (Avelar, Antunes, Santos, Andrade \& Dourado, 2008; Wright \& Vered, 2017). Identificado pelo seu crescimento lento e por ser localmente invasivo tem grande repercussão clínica em razão do seu caráter expansivo, além de representar cerca de 1\% a 3\% de todos os cistos e tumores odontogênicos (Navarro López, Lafuente, Falcón, Sassari Sandoval, \& Valdovinos Zaputovich, 2019; Neville et al., 2016).

De sintomatologia tardia, todas as variantes do ameloblastoma podem levar meses até serem identificadas pelo paciente ou pelo profissional de saúde (Kreppel \& Zöller, 2018), devido à apresentação geralmente assintomática em seus estágios iniciais. No entanto, sinais e sintomas podem estar presentes sejam eles má oclusão, deformidade facial, invasão dos tecidos moles, mobilidade dentária e a limitação do movimento mandibular (Wright \& Tekkesin, 2017). Assim como no caso relatado, muitas vezes, a principal característica é o aumento de volume causando deformidade facial, diminuição da autoestima e da qualidade de vida do paciente, o que geralmente só ocorre após a progressão da lesão (Kreppel \& Zöller, 2018). Essa realidade dificulta o diagnóstico precoce e culmina em um tratamento menos conservador e prognóstico menos favorável (Chae et al., 2015).

Em relação às características radiográficas, na variante multicística ou convencional é possível observar lesões radiolúcidas multiloculares, com bordas irregulares e padrão de bolhas de sabão ou favos de mel, causando abaulamento e diminuição de espessura da cortical óssea (Freire, Junior, Albuquerque Junior, \& Souza, 2021; Santana, Sila, \& Horiuchi, 2020; White \& Pharoah, 2019). Já o tipo unicístico apresenta radiolucência unilocular e geralmente bordas regulares. Nos dois padrões é comum observar reabsorção radicular das raízes próximas a lesão e em muitos casos um dente não erupcionado, geralmente o terceiro molar inferior, pode estar associado a área do tumor (Navarro López et al., 2019; Neville et al., 2016; Wright \& Tekkesin, 2017). 
Por possuírem características distintas, a literatura mostra que a variante convencional possui uma taxa de recidiva de $50 \%$ a $90 \%$ devido ao seu caráter infiltrativo enquanto que o ameloblastoma unicístico apenas de $10 \%$ a $20 \%$ (Avelar et al., 2008; Neville et al., 2016; White \& Pharoah, 2019). Portanto, a escolha de um tratamento mais radical com ressecção de margem de segurança dá um melhor prognóstico, porém a decisão do tratamento instituído não depende apenas do tipo histológico da lesão como também de fatores como a idade do paciente, o padrão clínico e radiográfico da lesão, o tempo de evolução, tratamento prévio realizado e morbidade gerada ao paciente (Hendra et al., 2019; Slusarenko et al., 2018). Por possuir a maior taxa de recidiva e pior prognóstico, a variante convencional é geralmente tratada de forma mais radical com ressecção da lesão com margem de segurança, enquanto o unicístico acomete pacientes jovens com mais frequência, é menos invasivo e tem mostrado bons resultados com terapias conservadoras. (Hendra et al., 2019; Moraes et al., 2014).

Dentre os tratamentos conservadores, a descompressão está indicada para lesões de aspecto cístico com o objetivo de diminuir o tamanho da lesão e tornar mais segura a enucleação e curetagem do tumor (Santana et al., 2019). Fatores como idade, apresentação clínica, bem estar do paciente e comprometimentos estéticos e funcionais devem ser levados em conta na hora da escolha do tratamento, em razão disso o tratamento conservador tem sido escolhido por otimizar a qualidade de vida do paciente e reduzir a necessidade de ressecção óssea, mesmo com a técnica radical apresentando altas taxas de sucesso (McClary et al., 2016).

No caso relatado, as características císticas observadas nos exames de imagem e através da punção aspirativa permitiram que fosse optado por um tratamento conservador inicial com o intuito de garantir uma menor morbidade da paciente. Apesar da descompressão não ter tido o sucesso esperado, a enucleação e curetagem foram suficientes para gerar uma reparação óssea devolvendo a paciente aspectos estéticos e funcionais satisfatórios. Independente do tratamento de escolha é importante destacar a necessidade de acompanhamento clínico e radiográfico dessas lesões por anos após o procedimento a fim de proservar o paciente pelo maior tempo possível.

\section{Conclusão}

As implicações estéticas e funcionais dos tumores odontogênicos possuem reflexo direto na qualidade de vida dos pacientes. Por isso, a escolha correta do tratamento deve-se atentar, principalmente, as características relacionadas ao tumor e ao paciente. $\mathrm{O}$ tratamento do ameloblastoma é amplamente discutido na literatura, porém não existe um consenso em relação ao procedimento mais indicado. Apesar da ressecção cirúrgica ser a opção de tratamento mais preconizada, estudos que visem o desenvolvimento de técnicas menos invasivas são necessários visto que um tratamento mais conservador aumenta a qualidade de vida do paciente. Independente do tratamento de escolha é importante destacar a necessidade de acompanhamento clínico e radiográfico dessas lesões por anos após o procedimento a fim de proservar o paciente pelo maior tempo possível.

\section{Referências}

Avelar, R. L., Antunes, A. A., Santos, T. S., Andrade, E. S. \& Dourado, E. (2008). Tumores odontogênicos: estudo clínico-patológico de 238 casos. Revista Brasileira de Otorrinolaringologia, 74(5), 668-673.

Chae, M. P., Smoll, N. R., Hunter-Smith, D. J., \& Rozen, W. M. (2015). Establishing the natural history and growth rate of ameloblastoma with implications for management: systematic review and meta-analysis. PloS one, 10(2), e0117241.

da Silva, L. P., Serpa, M. S., Tenório, J. R., do Nascimento, G. J., de Souza-Andrade, E. S., \& Veras-Sobral, A. P. (2016). Retrospective study of 289 odontogenic tumors in a Brazilian population. Medicina oral, patologia oral y cirugia bucal, 21(3), e271-e275.

Freire, R. T., Junior, A. R. P., Albuquerque Junior, R. L. C. V. \& Souza, L. M. A. (2021). Ameloblastoma em região anterior de mandíbula. Revista Cubana de Estomatologia, 58(1):e3028.

Hendra, F. N., Kalla, D. S. N., Cann, E. M. V., de Vet, H. C., Helder, M. N., \& Forouzanfar, T. (2019). Radical vs conservative treatment of intraosseous ameloblastoma: Systematic review and meta-analysis. Oral diseases, 25(7), 1683-1696. 
Research, Society and Development, v. 10, n. 4, e48110514077, 2021

(CC BY 4.0) | ISSN 2525-3409 | DOI: http://dx.doi.org/10.33448/rsd-v10i5.14077

Kreppel, M., \& Zöller, J. (2018). Ameloblastoma-Clinical, radiological, and therapeutic findings. Oral diseases, 24(1-2), $63-66$.

McClary, A. C., West, R. B., McClary, A. C., Pollack, J. R., Fischbein, N. J., Holsinger, C. F., Sunwoo, J., Colevas, A. D., \& Sirjani, D. (2016). Ameloblastoma: a clinical review and trends in management. European archives of oto-rhino-laryngology: official journal of the European Federation of Oto-Rhino-La ryngological Societies (EUFOS): affiliated with the German Society for Oto-Rhino-Laryngology - Head and Neck Surgery, 273(7), 1649-1661.

Moraes, F. B., Cardoso, R. M. N., Rodrigues, S. V., Dutra, M. V. F., Pereira, U. R., \& Borges, T. R. S. A. (2014). Ameloblastoma: uma análise clínica e terapêutica de seis casos. Revista Brasileira de Ortopedia, 49(3), 305-308.

Navarro López, J. S. A., Lafuente, A. C., Falcón, D. E., Sassari Sandoval, M. G., \& Valdovinos Zaputovich, B. M. (2019). Ameloblastoma: caso clínico patológico en pediatría. Revista del Ateneo Argentino de Odontologia, 61(2): 8-12.

Neville, B. W., Damm, D. D., Allen, C. M., \& Bouquot, J. E. (2016). Patologia Oral e Maxilofacial. (4a ed.). Elsevier.

Rioux-Forker, D., Deziel, A. C., Williams, L. S., \& Muzaffar, A. R. (2019). Odontogenic Cysts and Tumors. Annals of plastic surgery, 82(4), $469-477$.

Santana, K. M., Sila, R. P. \& Horiuchi, N. C. F. N. (2020). Ameloblastoma e suas características clínicas e radiográficas: relato de caso clínico. Revista Odontológica de Araçatuba, 40(2):48-53.

Santana, T. C. A., Câncio A. V., Ramos T. C. F., Hassam S. F., Cardoso J. A. \& Farias J.G. (2019). Ameloblastoma unicístico: relato de caso. Revista Da Faculdade De Odontologia - UPF, 24(2):284-91.

Santosh, A. B. R, \& Ogle, O. E. (2020). Odontogenic Tumors. Dental clinics of North America, 64(1), 121-138.

Shi, H. A., Ng, C. W., Kwa, C. T., \& Sim, Q. X. (2020). Ameloblastoma: A succinct review of the classification, genetic understanding and novel molecular targeted therapies. The surgeon: journal of the Royal Colleges of Surgeons of Edinburgh and Ireland, S1479-666X(20)30081-0.

Slusarenko da Silva, Y., Tartaroti, N. A., Sendyk, D. I., Deboni, M., \& Naclério-Homem, M. (2018). Is conservative surgery a better choice for the solid/multicystic ameloblastoma than radical surgery regarding recurrence? A systematic review. Oral and maxillofacial surgery, 22(4), 349-356.

White S., Pharoah M. (2019). Radiologia oral de White e Pharoah: princípios e interpretação. (8a ed.). Elsevier.

Wright, J. M., \& Tekkesin, M. S., (2017). Odontogenic tumors: where are we in 2017? Journal of Istanbul University Faculty of Dentistry, 51(3), S10-S30.

Wright, J. M., \& Vered, M. (2017). Update from the 4th Edition of the World Health Organization Classification of Head and Neck Tumours: Odontogenic and Maxillofacial Bone Tumors. Head and neck pathology, 11(1), 68-77.

de Medeiros, W. K., da Silva, L. P., Santos, P. P., Pinto, L. P., \& de Souza, L. B. (2018). Clinicopathological analysis of odontogenic tumors over 22 years period: Experience of a single center in northeastern Brazil. Medicina oral, patologia oral y cirugia bucal, 23(6), e664-e671. 